\title{
¿ERA LA INDUSTRIA ELÉCTRICA DE ENTREGUERRAS UN MONOPOLIO NATURAL? EVIDENCIA A PARTIR DE LA SOCIEDAD HIDROELÉCTRICA ESPAÑOLA*
}

\author{
ANNA MARIAAUBANELL JUBANY \\ Universitat Autònoma de Barcelona ${ }^{\mathrm{a}}$
}

\section{RESUMEN}

En los últimos años se ha cuestionado la condición de monopolio natural de la industria eléctrica. La fase de generación ya no es considerada como un monopolio natural, y se debate que la fase de distribución lo sea. Este trabajo parte de las principales aportaciones que se han realizado desde la teoría industrial en vistas a su aplicación a la Sociedad Hidroeléctrica Española, como empresa significativa de la industria eléctrica española del período de entreguerras. El resultado del análisis ha confirmado el carácter de monopolio natural de cada una de las fases de generación, transporte y distribución. Dado que la industria eléctrica del período de entreguerras presentaba una estructura monopolística, estos resultados indican que era el tipo de organización óptima.

Palabras clave: monopolio natural, empresa eléctrica, economías de escala, 19141935

* Agradezco el interés y ayuda de Walter García-Fontes y los valiosos comentarios de tres evaluadores. Los errores son de mi exclusiva responsabilidad.

a Departament d'Economia i Història Econòmica, Edifici B, 08193 Bellaterra (Cerdanyola del Vallès), Barcelona. anna.aubanell@uab.es 


\begin{abstract}
Over the past two decades it has been questioned whether the electric utility industry is a natural monopoly. Electricity generation is no longer considered a natural monopoly and the topic is debated in relation to electricity distribution. This article explores the main contributions in this field in order to apply their findings to the Spanish Electric Utility Industry in the inter-war period. The study focuses on one of the major Spanish companies, Sociedad Hidroeléctrica Española, and shows that generation, transmission and distribution of electricity were natural monopolies. The results indicate that the monopolistic structure which the inter-war electric utility industry exhibited was the optimal form of organisation.
\end{abstract}

Keywords: natural monopoly, electric utilities, scale economies, interwar, Spain JEL Classification: N74, L94, D42

\title{
1. INTRODUCCIÓN
}

Hasta la década de los ochenta la condición de monopolio natural de industrias como la eléctrica o las telecomunicaciones era generalmente aceptada. Esta aceptación de la condición de monopolio natural había impulsado la nacionalización de buena parte de dichas industrias en Europa después de la Segunda Guerra Mundial, surgiendo monopolios de ámbito nacional. En los países donde no nacionalizaron las empresas los gobiernos las regularon para evitar la pérdida de bienestar que tiene lugar cuando los precios y la producción responden a criterios monopolísticos.

Al final de los años setenta aparecieron varios trabajos que sentaron un nuevo marco teórico formal para el estudio del monopolio natural. Entre ellos cabe resaltar el artículo de Baumol sobre los tests más adecuados para determinar la condición de monopolio natural de una industria ${ }^{1}$. Los trabajos empíricos que se realizaron desestimaron que la fase de generación de la industria eléctrica tuviera el carácter de monopolio natural, y se empezó a cuestionar el que la fase de distribución lo fuera. De especial impacto fue el artículo de Christensen y Greene (1976), que estimaron las economías de escala de la fase de generación de la industria eléctrica norteamericana demostrando que estaban presentes en 1975. Replicando la estimación de Nerlove (1963) corroboraron que las economías de escala existían en 1955. Por lo que se destacaba la temporalidad de la condición de monopolio natural de la industria eléctrica. Estos trabajos han inducido a la pregunta: ¿era la industria eléctrica de entreguerras un monopolio natural?

${ }^{1}$ Baumol, (1977, pp. 809-822). En este ámbito, cabe destacar el trabajo de Sharkey (1982). 
Determinar la condición de monopolio natural de una industria resulta imprescindible para conocer cuál es la mejor organización de la producción. Si una industria es monopolio natural, la forma óptima de organización es el monopolio. Los inconvenientes son que la empresa tenderá a vender a precios de monopolio, a ser ineficiente en la producción y a realizar un servicio de escasa calidad. Por una parte, esta práctica perjudica a los consumidores y, por otra, puede afectar la eficiencia del mercado, ya que los elevados beneficios estimulan la entrada en el mercado de nuevas empresas, generando competencia que crea ineficiencia. Estos dos hechos hacen que sea necesaria la introducción de controles de precios y/o de entrada, es decir, la regulación del mercado por parte de la administración pública o por las propias empresas. En caso de no presentar las condiciones de monopolio natural la situación de competencia permite la mejor asignación de recursos.

La industria eléctrica en España tendió a la monopolización a medida que aumentó la importancia de la energía hidroeléctrica. Varios autores han señalado este hecho y el impacto negativo causado por el poder monopolístico de las grandes empresas ${ }^{2}$. Este artículo tiene por objetivo conocer el carácter de monopolio natural de cada una de las fases de la industria eléctrica del período de entreguerras para establecer si la organización monopolística del mercado fue la óptima. En caso de ser la organización óptima, se deberá estudiar si la regulación ejercida por las administraciones públicas fue suficientemente eficiente como para limitar las pérdidas de bienestar derivadas del comportamiento monopolístico. Pero éste será el objetivo de un próximo trabajo.

A continuación se presenta la teoría de monopolio natural y se explora su aplicación a la industria eléctrica considerando las características específicas de ésta. Le seguirá un apartado dedicado a analizar algunas de las fuentes de monopolio natural de la industria eléctrica, para lo que se utiliza la información disponible de las empresas eléctricas ubicadas en Madrid antes de la Guerra Civil. Por último, se estimarán las funciones de costes de cada fase de producción para determinar la condición de monopolio natural de la industria eléctrica en el período de entreguerras a partir del estudio de Hidroeléctrica Española.

\section{LA TEORÍA DEL MONOPOLIO NATURAL Y LA ESPECIFICIDAD DE LA IN- DUSTRIA ELÉCTRICA}

Baumol define monopolio natural como aquella situación en la que una empresa genera un abanico de niveles de producción al menor coste social. A diferencia de la literatura tradicional, que subrayaba la importancia de las economías de es-

${ }^{2}$ Antolín (1999) y Sudrià (1990). Para Antolín, el poder monopolístico de las empresas es el factor causante del bajo consumo de electricidad por habitante en España. 
cala a la hora de establecer qué era un monopolio natural, para Baumol las economías de escala no son condición necesaria ni suficiente para que exista un monopolio natural ${ }^{3}$. El concepto clave es el de la subaditividad de la función de costes. Esto es, la suma de los costes de cualquier vector de niveles de producción tiene que ser menor que la suma de los costes de producirlos por separado ${ }^{4}$.

A primera vista, la noción es sencilla, pero para probar la existencia de subaditividad es necesario conocer la forma de toda la función de costes desde el origen hasta el output concreto. Empíricamente esto es imposible, ya que son necesarios datos que no se encuentran disponibles a través de la información que facilitan las empresas, pues sus datos se refieren a una parte de la función de costes.

El modelo de Baumol presenta dos restricciones: la inexistencia de cambio tecnológico y la disponibilidad de la tecnología existente tanto para el monopolista como para cada uno de los potenciales competidores. Así pues, todos los precios de los factores son fijos. Esta restricción implica que la condición de subaditividad tiene un carácter temporal. Cuando una industria presenta una curva de costes que es subaditiva lo que indica es que esta industria es un monopolio natural en ese momento del tiempo, porque presenta los menores costes dados los precios.

Si bien la condición de subaditividad es empíricamente difícil de demostrar, Baumol demuestra que, para las empresas que generan un único producto, la existencia de economías de escala es siempre condición suficiente, aunque no necesaria, para la subaditividad. No es necesaria porque la función de costes puede ser subaditiva cuando han dejado de existir economías de escala debido a que la demanda no es lo suficientemente elevada para que dos empresas produzcan de forma eficiente. Para aquellas empresas que producen más de un bien, la presencia de economías de escala no es condición suficiente. El hecho de que la existencia de economías de escala sea una condición suficiente cuando se produce un solo producto tiene una gran importancia para este artículo, ya que es relativamente fácil conocer las economías de escala de una empresa. Así pues cabe discutir si la industria eléctrica genera uno o varios productos.

En la década de los ochenta, a la vez que se cuestionaba la condición de monopolio natural de la industria también se cuestionaba que la unidad de análisis fuera la empresa integrada verticalmente. La idea es que la empresa eléctrica en su conjunto puede ser un monopolio natural, pero cada una de las fases del proceso de producción no tiene por qué serlo. Cada fase tiene una tecnología particular que genera una función de costes diferente. De ello se desprende que es necesario estudiar las fases de generación, transporte y distribución de electricidad por separado y así conocer exactamente la fase o fases que son monopolio natural. En el caso de que una fase no tenga las características de monopolio natural,

\footnotetext{
${ }^{3}$ Este apartado está basado en gran medida en el artículo de Baumol (1977, pp. 809-811).

${ }^{4}$ Subaditividad: $\mathrm{C}(\mathrm{q})<\mathrm{C}\left(\mathrm{q}_{1}\right)+\ldots+\mathrm{C}\left(\mathrm{q}_{\mathrm{m}}\right)$
} 
la introducción de la competencia en dicha fase es la solución al comportamiento monopolístico que puede existir.

Partiendo de la necesidad de analizar las fases por separado, hace falta discutir si la empresa genera uno o varios productos por fase. Si elabora un único producto, la existencia de economías de escala prueba que dicha fase tiene las características de un monopolio natural.

En la fase de generación existen tantos productos como combustibles primarios se usen para producir electricidad. En la actualidad, las principales fuentes de energía primaria son carbón, petróleo, gas natural, nuclear, hidráulica, eólica y solar. En cambio, en la fase de transporte solamente se genera un único producto.

La fase de distribución cuenta con varios productos. Por un lado, los kilovatios servidos a diferentes voltajes se pueden considerar como productos diferentes. La existencia de una red de alta tensión -en realidad es de media tensión- y otra de baja tensión indica que se generan dos productos diferentes: kilovatios a alto y kilovatios a bajo voltaje. El coste de distribuir el kilovatio a baja tensión es mayor porque las pérdidas de transmisión son mayores cuanto menor es el voltaje, lo que justificaría la definición de dos productos. Los trabajos de Joskow y Schmalensee (1983), Roberts (1986) y Thompson (1997) consideran que se generan estos dos productos, aunque por falta de los datos necesarios se ven forzados a identificar los consumidores de alta tensión con los abonados industriales y los de baja tensión con los abonados comerciales y domésticos. En el caso de disponer de los costes desagregados para cada red, la solución es considerar la distribución a alta y baja tensión por separado, de forma que cada una produce un único producto.

Salvanes y Tjotta (1998) también consideran que en la fase de distribución se obtienen dos productos. Uno es el número de abonados, en el sentido de que, al firmar el contrato de suministro, el consumidor adquiere la opción de comprar electricidad en su casa. El otro producto es la electricidad consumida. Si bien esta aproximación resulta apropiada teniendo en cuenta la tarificación actual, tiene poco sentido aplicarla en el período de entreguerras, en el que existían contratos a tanto alzado, y en muchos casos ni siquiera se pagaba por el alquiler del contador.

Por último, se puede considerar que existen tantos productos como tarifas que generen costes diferentes. En estas dos últimas interpretaciones, la presencia de las economías de escala no es suficiente para determinar la existencia de monopolio natural, porque se generan dos o más productos.

\section{FUENTES DE MONOPOLIO NATURAL}

A continuación se exponen los diferentes conceptos que recogen las características de la industria eléctrica susceptibles de generar las condiciones de mono- 
polio natural. Entre los más comúnmente citados se encuentran las economías de escala, las economías de densidad y tamaño y, por último, se considera el papel de las externalidades generadas por las empresas.

\subsection{Economías de escala}

La capacidad por parte de una única empresa de producir a un coste menor que varias empresas puede ser debida a la existencia de economías de escala. En presencia de economías de escala la curva de costes medios a largo plazo tiene una pendiente negativa.

Entre los factores que generan las economías de escala, las elevadas inversiones han centrado la atención de los economistas desde las primeras formulaciones de la teoría de monopolio natural. En efecto, las empresas eléctricas, y más aún las hidroeléctricas, necesitan elevadas inversiones de capital inicial en comparación con sus ingresos de explotación. Un aumento de la producción permite que el coste medio disminuya, ya que se reparte entre un mayor número de unidades producidas. El indicador más comúnmente utilizado para conocer si una industria es capital intensiva es la ratio activo inmovilizado/ingresos. La industria eléctrica tiene una ratio bastante más elevada que otras industrias, siendo ésta una causa de que los costes medios sean decrecientes.

En el cuadro 1 se relacionan los cocientes de activo inmovilizado/ingresos para algunas empresas eléctricas españolas en 1930. Adicionalmente se ha calculado el cociente activo neto/ingresos para poder realizar una comparación con empresas ferroviarias debido a que no se dispone del inmovilizado de éstas. Los datos son, cuando menos, interesantes. Los cocientes inmovilizado/ingresos de las empresas eléctricas son muy diferentes entre sí. La ratio de la Sociedad Hidroeléctrica Española era 8,71, la de Unión Eléctrica Madrileña era tres puntos menor -5,63- y la de la Cooperativa Electra Madrid dos veces y media inferior a la de la primera y dos puntos inferior a la de la segunda.

El cociente de la Sociedad Hidroeléctrica Española puede estar ligeramente sobrevaluado a causa de la disminución de ingresos $-4,3$ por cien- provocada por la competencia en el mercado de Valencia. Sin embargo, esto no justifica las grandes diferencias entre las tres eléctricas. La explicación procede del diferente peso que tenían las fases de generación, transporte y distribución en las empresas. La Sociedad Hidroeléctrica Española se dedicaba a generar hidroelectricidad, a transportarla y distribuirla a alta tensión. La distribución a baja tensión la realizaban otras empresas como la Cooperativa Electra Madrid. La Unión Eléctrica Madrileña integraba todas las fases de producción, si bien una gran parte de la electricidad la adquiría de sus filiales productoras. La baja ratio de Cooperativa, que era una empresa exclusivamente distribuidora a baja tensión, indica que la 


\section{CUADRO 1}

RATIOS INMOVILIZADO/INGRESOS Y ACTIVO NETO/INGRESOS (1930)

\begin{tabular}{|l|c|c|}
\hline & Inmovilizado/ingresos & Activo neto/ingresos \\
\hline S. Hidroeléctrica Española & 8,71 & 9,54 \\
\hline Unión Eléctrica Madrileña & 5,63 & 6,99 \\
\hline Cooperativa Electra Madrid & 3,46 & 4,06 \\
\hline MZA & & 7,36 \\
\hline Norte & & 6,20 \\
\hline
\end{tabular}

Fuentes: Sociedad Hidroeléctrica Española, Memoria 1930; Unión Eléctrica Madrileña, Memoria 1930; Anuario Financiero y de Sociedades Anónimas, 1931, y A. Carreras y X. Tafunell (1993), p. 168.

fase de distribución era la menos capital intensiva, en relación con generación y transporte. Este hecho corrobora el menor cociente de Unión en relación al de Hidroeléctrica Española. Las fases más capital intensivas eran generación y transporte, lo cual no es de extrañar porque en 1930 estas empresas generaban principalmente hidroelectricidad.

Las ratios de las empresas ferroviarias eran inferiores a las de las eléctricas. En cambio, si se compara con los datos americanos de 1962 utilizados por Primeaux, los ferrocarriles tienen unos cocientes superiores a las eléctricas -4,63 y 3,54 respectivamente $^{5}$. Es posible que ello se derive del mayor peso de la energía termoeléctrica en la industria americana, lo que también explicaría, en parte, por qué los cocientes de las eléctricas españolas son mayores que los americanos ${ }^{6}$. El capital necesario para las instalaciones térmicas era inferior al de las instalaciones hidroeléctricas. En 1914, el coste de la central térmica de Madrid era de 294 pesetas por kilovatio instalado, y el coste de la central hidroeléctrica del Molinar era de $340 \mathrm{ptas} / \mathrm{kW}$. Si se añade el coste de la línea de transporte, el coste asciende a 877 ptas/kW. En otras palabras: el capital invertido en la instalación hidroeléctrica -central más línea de transporte- era tres veces mayor al destinado a la central térmica.

La principal crítica que se ha realizado al uso de la ratio activo inmovilizado/ ingresos es que no tiene en cuenta el grado de depreciación del capital invertido. El capital invertido en la construcción de una presa para regular el aforo del río tiene una vida mucho más larga que el capital invertido en la compra de una máquina de vapor. De esta manera, el coste de las grandes cantidades de capital

\footnotetext{
${ }^{5}$ Primeaux (1979, p. 70).

${ }^{6}$ En 1930 el 65 por cien de la producción de electricidad en Estados Unidos procedía de centrales térmicas y el 34 por cien de centrales hidroeléctricas. En 1950 los porcentajes eran 70 y 29 respectivamente.
} 
invertido debería ponderarse por la depreciación. Como el capital invertido en la industria eléctrica tiene una vida media superior a la de otras industrias, el uso de la ratio sin introducir ningún tipo de corrección por el grado de depreciación no resulta adecuado.

Aun aceptando que las empresas hidroeléctricas no fueran tan capital intensivas si se introdujera una corrección según la vida del capital invertido, las grandes sumas de capital requeridas generan una serie de costes que pueden ser susceptibles de inducir economías de escala. Garfield y Lovejoy señalan que las grandes cantidades de capital que necesitan las eléctricas acarrean «unos mayores costes debido a mayores intereses, dividendos, impuestos, seguros y amortizaciones que en empresas con menores necesidades de capital» ${ }^{7}$. Independientemente del grado de amortización del capital, la factura a pagar anualmente por esas elevadas sumas aumenta los costes fijos, y al aumentar la producción los costes medios decrecen, lo que apoya la idea de que las empresas con gran cantidad de capital son susceptibles de ser monopolio natural.

Por lo que respecta a las eléctricas arriba consideradas, los costes de capital fueron importantes durante el período estudiado. Entre 1914 y 1935, el capital ajeno formado por títulos de renta fija ascendía a un 45 por cien del capital nominal, entendido como acciones más obligaciones. En varios años las obligaciones llegaron a representar el 59 por cien del capital nominal. Los intereses pagados a los obligacionistas ascendían al 30 por cien de los costes totales -gastos de explotación más cargas sociales-, llegando en el caso de Hidroeléctrica Española a alcanzar el 53 por cien en 1928.

Si al coste del capital ajeno se añade el coste del capital propio definido como el monto de dividendos repartidos, el argumento adquiere mayor peso. El coste del capital -propio y ajeno- suponía el 55 por cien de los costes totales -gastos de explotación más el coste del capital fijo y ajeno. Para Hidroeléctrica Española, el coste del capital llegó a representar el 77 por cien de los costes totales en 1928.

En resumen, las altas sumas de capital invertido en la industria eléctrica generan unos costes fijos elevados, siendo susceptibles de provocar una disminución de los costes medios. Dado que aparecen suficientes indicios de la existencia de economías de escala, aunque sean a nivel de la empresa integrada verticalmente, se considera oportuno estimar las funciones de costes necesarias para conocer si existieron economías de escala. Este ejercicio se realiza en el apartado 4.

\subsection{Economías de densidad y tamaño}

Dadas las particulares características de los negocios de red se ha refinado el concepto de economías de escala para conocer cuáles son los factores que, al

${ }^{7}$ Garfield y Lovejoy, (1964, p. 17). 
aumentar la producción, hacen disminuir el coste medio. El concepto de economías de densidad se refiere a la evolución de los costes medios cuando aumenta la producción manteniendo constantes el número de abonados y el área geográfica.

Los trabajos de Weiss (1975) y Joskow y Schmalensee (1983) señalaron la importancia de la densidad de la demanda, entendida como la cantidad demandada dentro de un área geográfica, como factor determinante de las economías de escala. Roberts (1986) matizó la correlación entre densidad de la demanda y disminución del coste medio. Un aumento de la densidad de la demanda puede deberse a diferentes factores que tienen impactos diversos sobre la función de costes. Así, las economías de densidad se dividen en economías de densidad de producto y economías de densidad de abonado.

Las economías de densidad de producto existen cuando un incremento de la cantidad demandada por los abonados de una empresa, manteniendo constante el número de abonados y el tamaño de la red, lleva a una disminución del coste medio. Es presumible que un aumento de los kilovatios consumidos por abonado lleve siempre a una disminución del coste medio.

Las economías de densidad de abonados se dan cuando un incremento de la demanda debido a un aumento del número de abonados, sin variar el consumo por abonado ni la extensión de la red, genera una disminución del coste medio. A diferencia del aumento de la densidad de producto, no siempre un aumento de la densidad de abonados lleva a la disminución del coste medio. Esto es debido a que la disminución del coste medio a causa del aumento de la electricidad distribuida puede ser absorbida por el aumento de los costes provocados por el aumento de abonados.

Las economías de tamaño existen cuando un aumento de la demanda a causa de la expansión de la red de distribución provoca una disminución del coste medio. Al igual que en el caso anterior, la presencia de economías de tamaño es menos probable porque la expansión de la red implica un aumento del capital fijo, de forma que la disminución del coste medio ocasionada por el aumento de la producción puede quedar neutralizada por el aumento del coste del capital fijo.

La presencia de economías de densidad implica que la mejor forma de suministrar a un abonado es a través de una única empresa. Cuando no existen economías de densidad o éstas se han agotado, la presencia de dos compañías en la misma zona puede beneficiar a los consumidores, ya que la disminución de la densidad no conlleva un aumento del coste medio y en cambio puede generar una competencia que comporte una disminución de precios.

La mayoría de los trabajos empíricos muestran la presencia de economías de densidad, siendo su principal fuente el aumento de la densidad de producto y, en mucha menor medida, el aumento de la densidad de abonado ${ }^{8}$.

${ }^{8}$ Roberts (1986), Nelson y Primeaux (1988) y Thompson (1997). 
En este caso, desafortunadamente, no se puede estimar de forma apropiada una función de costes que permita el análisis de las economías de densidad y tamaño, porque con los datos disponibles se consiguen diecinueve observaciones y los coeficientes a estimar ascienden a veintiocho. Esto impide conocer cuál es la contribución de las economías de densidad de producto, de las de densidad de abonado y de las de tamaño a la disminución de los costes medios.

A modo de aproximación y según los datos de la Cooperativa Electra Madrid, entre 1914 y 1932 se produjo un aumento de la densidad de producto cuando el consumo por abonado pasó de 18 a 26 kWh al mes. Por tanto, sería de esperar que existieran economías de densidad de producto en la distribución a baja tensión en el período considerado. Otra cuestión es si estas economías quedaron neutralizadas por el aumento de los costes que pudieron generar el incremento del número de abonados o la extensión del tamaño de la red.

\subsection{Externalidades negativas}

Entre las condiciones que se han propuesto para la existencia de monopolio natural que no están directamente relacionadas con las economías de escala y de densidad cabe destacar los inconvenientes para los consumidores que se derivan de la existencia de más de una empresa. No se consideran en este estudio las externalidades negativas de la construcción de las centrales hidroeléctricas -principalmente ecológicas- ni de las térmicas -ecológicas y sanitarias-, ya que están más vinculadas a la cantidad producida que al número de empresas en el mercado. Las externalidades negativas aquí tratadas son las de la fase de distribución de electricidad cuando éstas pueden aumentar con el número de empresas presentes en el mercado.

Caywood y Carves (1956) consideraron como condición de monopolio natural los inconvenientes experimentados por los ciudadanos debido a las frecuentes reparaciones llevadas a cabo por las empresas que extienden sus redes en una misma zona. Según Primeaux, la duplicidad de redes no supone inconveniente alguno para el ciudadano, y la competencia reduce la duración de las reparaciones. Este autor basa su argumento en un estudio sobre duopolios americanos en la década de los sesenta. La primera observación a hacer al estudio de Primeaux es que no explica cómo mide la existencia o inexistencia de los inconvenientes sufridos por los ciudadanos.

Las molestias causadas por las reparaciones dependen de la densidad de población urbana. Aun aceptando que Primeaux no encontrara inconvenientes a la duplicidad de redes, la baja densidad y el sistema de amplias vías de comunicación de una ciudad de tamaño mediano americano podrían explicar tal resultado, que no podría extenderse a ciudades de alta densidad y congestión como las medianas y 
grandes ciudades europeas. Por un lado, la arquitectura predominantemente horizontal en el tipo de ciudades de su estudio es diametralmente opuesta a la presente en la mayoría de las ciudades europeas mencionadas, donde predomina la verticalidad. Además, los núcleos de las viejas ciudades europeas presentan un sistema de calles estrechas que aumentan las molestias. Por lo tanto, al tener una mayor densidad aumenta el número de posibles afectados; la escasa amplitud de las calles hace que simples reparaciones obliguen muchas veces a cortar el tráfico o, en todo caso, a crear problemas de fluidez vial. Esto sin considerar otros muchos inconvenientes como los ruidos, el precario acceso a las viviendas, el aumento de los accidentes, etc. Por otro lado, un efecto positivo sería el aumento de los ingresos municipales gracias a la concesión de licencias de apertura de zanjas.

La menor duración de las reparaciones argumentada por Primeaux a consecuencia de la competencia no es un buen indicador de las molestias sufridas por los ciudadanos a causa de la duplicidad de redes. El tiempo total en reparaciones por kilómetro de línea permitiría comparar los efectos de la duplicidad de líneas mucho más adecuadamente.

No existen datos sobre los tiempos de reparación para el período estudiado. En el caso de Madrid, las molestias sufridas por los ciudadanos debieron ser particularmente elevadas, ya que desde principios del siglo XX hasta 1913 se dieron varios períodos de competencia más o menos intensa entre las compañías eléctricas, produciéndose una superposición de redes de distribución ${ }^{9}$. En determinadas calles del centro de la ciudad pasaban hasta cinco líneas de diferentes empresas. La prensa local se hizo eco de las múltiples quejas de que eran objeto las compañías eléctricas debido tanto a las obras de extensión de sus redes como a las reparaciones de mantenimiento necesarias.

Medir las externalidades negativas siempre es difícil, y más aún en el pasado. Pero parece evidente que la duplicidad de redes aumenta las reparaciones por metro de calle y, en consecuencia, las molestias a los vecinos y ciudadanos. Otra cuestión es si este hecho puede considerarse como condición suficiente de monopolio natural. En cualquier caso, es un elemento a tener en cuenta que es ignorado por la teoría vigente de monopolio natural.

\section{ANÁLISIS DE MONOPOLIO NATURAL: LA SOCIEDAD HIDROELÉCTRICA ESPAÑOLA}

En páginas precedentes se ha mostrado que existen suficientes elementos para pensar que las empresas eléctricas presentan las características propias para ser un monopolio natural. En el apartado 2 se estableció el procedimiento para cono-

\footnotetext{
${ }^{9}$ Aubanell (1992).
} 
cer, según la teoría económica vigente, si una empresa presenta las características de monopolio natural a través del estudio de las economías de escala. Al mismo tiempo se señaló la necesidad imperativa de estudiar cada fase por separado. Esto significa estimar una función de costes para las fases de generación, transporte y distribución, por lo que se necesitan los datos de los costes totales -capital, personal, explotación y conservación- además de la cantidad producida y los precios de los factores -capital y trabajo- desagregados para cada fase.

Las escasas estadísticas de la industria española para el período de entreguerras no contienen la información que se precisa. Las memorias empresariales y los anuarios permiten conocer el coste total; de hecho, proporcionan el coste en la cuenta de pérdidas y ganancias, al que solamente hay que añadir el coste del capital propio obtenido a partir del activo y los dividendos. En todo caso, se trata de datos agregados que comprenden desde los costes de generación a los de distribución, pues las eléctricas, en su mayoría, eran empresas integradas verticalmente.

La única opción disponible para obtener la información desagregada es acudir a los archivos de las empresas. Afortunadamente, el Archivo de Iberdrola en Alcántara ha abierto sus puertas recientemente ${ }^{10}$. Este archivo contiene la documentación de la Sociedad Hidroeléctrica Española, sus filiales y las empresas por ella absorbidas. Esta razón, junto al hecho de que la organización empresarial de la compañía en el período considerado ofrece una clara separación entre la fase de distribución a baja y a alta tensión, justifican que el estudio de monopolio natural se base en dicha empresa. Además, la tecnología utilizada por Hidroeléctrica Española era similar a la utilizada por el resto de las empresas eléctricas españolas, por lo que los resultados obtenidos son extrapolables al resto de la industria.

La mayoría de los trabajos empíricos estudian las economías de escala con datos de corte transversal, considerando el conjunto de empresas de un país en un año determinado. La falta de datos impide la utilización de esta opción. La alternativa consiste en estimar la existencia de economías de escala a lo largo de un período, que ha sido el método utilizado en los casos en que la industria eléctrica ha sido nacionalizada y existe una sola empresa. El principal problema se encuentra en el posible cambio tecnológico. En este caso, se estaría computando a economías de escala las economías de la mejora tecnológica. Por lo tanto, es necesario conocer si se produjeron cambios tecnológicos que puedan invalidar los resultados.

\subsection{La empresa eléctrica de entreguerras: Hidroeléctrica Española y Coopera- tiva Electra Madrid}

A continuación, se exponen las características de cada una de las fases que son objeto de análisis. Hidroeléctrica Española se dedicaba a la generación, trans-

${ }^{10}$ Agradezco la atención y ayuda de Juan Carlos García Adán, su archivero. 
porte y distribución a alta tensión, mientras que la distribución a baja tensión la realizaban otras empresas que podían ser o no sus filiales ${ }^{11}$. En este estudio se ha escogido a la filial Cooperativa Electra Madrid como distribuidora de baja tensión.

Hidroeléctrica Española generaba hidroelectricidad y termoelectricidad. La estrategia productiva de la compañía era la producción hidroeléctrica, como el nombre de la razón social indica. Las centrales hidroeléctricas del período estudiado estaban situadas en los ríos Júcar y Cabriel. Las centrales térmicas, situadas en los principales centros de consumo, eran centrales auxiliares que servían para cubrir las caídas de producción durante los estiajes o los accidentes que se produjeran tanto en las centrales hidroeléctricas como en las líneas de transporte de la energía hidroeléctrica ${ }^{12}$. En ningún caso la estrategia productiva de la empresa pasaba por la utilización de la termoelectricidad de manera regular, como lo muestra el hecho de que dicha energía no superó de media el 4,8 por cien de la producción total de Hidroeléctrica Española entre 1912 y 1935. La estrategia hidroeléctrica respondía al diferencial de costes entre la producción hidroeléctrica y la térmica. El coste medio del kilovatio hora hidroeléctrico para el período 1914 a 1935 fue de 0,0243 pesetas, mientras que el coste más bajo alcanzado por el kilovatio térmico fue de 0,0758 pesetas, obtenido en 1932, cuando se alcanzó la máxima eficiencia en la nueva central térmica de Madrid al producir 27,5 millones de kilovatios ${ }^{13}$. En otras palabras, el coste del kilovatio hora térmico era tres veces mayor que el hidroeléctrico.

Hidroeléctrica Española minimizaba la función de costes al utilizar al máximo las centrales hidroeléctricas y al mínimo las térmicas. Por esta razón, y dada la escasa magnitud de la producción térmica, en el texto principal se ha considerado únicamente la generación de hidroelectricidad. Gracias a que los datos de generación son desagregados para las dos tecnologías, se han estimado las dos funciones de costes. La estimación de la función de costes de la generación térmica se ha desplazado al apéndice en aras de una exposición menos iterativa. Así, la estimación de las economías de escala a través de la función de costes de producción hidroeléctrica y térmica permitirá saber si la fase de generación era un monopolio natural debido a que se trata de un único producto en cada caso. Durante el período estudiado no existen cambios tecnológicos destacables, por lo que, al estimar la función de costes de la generación hidroeléctrica entre 1914 y 1935, se obtendrá una correcta estimación de las economías de escala.

Una vez generada la hidroelectricidad hace falta transportarla hasta los centros de consumo. Las pérdidas debidas al transporte disminuyen al aumentar el

${ }^{11}$ Para un análisis de la estrategia empresarial de Hidroeléctrica Española véase Tedde (1987), Aubanell (2000) y Cayón (2002).

${ }^{12}$ Hidroeléctrica Española tenía centrales térmicas en Madrid, Valencia y Cartagena.

${ }^{13}$ El cálculo del coste del kilovatio hora hidroeléctrico se ha realizado a partir de los costes totales de generación hidroeléctrica más los correspondientes a transporte y recepcióntransformación dividido por la hidroelectricidad generada menos las pérdidas en transporte. 
voltaje, por lo que en la central generadora se eleva el voltaje para transportar la electricidad hasta el centro de consumo, donde en la central receptoratransformadora se disminuye el voltaje. Según las leyes de la física, la potencia transmitida crece en función del cuadrado de la tensión. Lo lógico sería emplear el voltaje más elevado posible porque las pérdidas serían mínimas, pero cuanto más elevada es la tensión más caro el material necesario. O sea, la empresa decide teniendo en cuenta la cantidad a transportar, la distancia y el coste de instalación.

La red de transporte de Hidroeléctrica Española en 1914 unía las centrales hidroeléctricas con los principales centros de consumo. De la central del Molinar partía una línea de 66.000 voltios hacia Madrid que, en Olmedilla, conectaba con la línea procedente de la central de Villora. En la central del Molinar salían dos líneas en dirección Este, una a Valencia y la otra a Alcoy, donde proseguía a un voltaje menor hasta Cartagena. La red de transporte se extendió a medida que aumentaba el consumo y los centros abastecidos. Así, diez años después, en 1924, se construyó una línea que conectaba directamente Molinar y Cartagena y una nueva línea a 132.000 voltios entre Molinar y Madrid. La tecnología utilizada en la producción de los cables de 132.000 y 66.000 voltios era la misma y presentaba el mismo problema de estrés en paralelo. La solución técnica solamente llegó en 1928, dando lugar a los cables tipo $\mathrm{H}^{14}$. En suma, la estimación de las economías de escala será la correcta porque no existió cambio técnico.

La fase de distribución está compuesta por redes de distribución de alta -en realidad es distribución a media tensión- y redes de distribución a baja. Gran parte de las empresas distribuidoras realizan ambas actividades, lo que ha llevado a la mayoría de autores que han realizado estimaciones de la función de costes de la distribución de electricidad a considerar que existen dos productos: electricidad a alta y electricidad a baja. Si se obtienen dos productos en distribución, la presencia de economías de escala no es condición suficiente para probar que es un monopolio natural.

Afortunadamente, la organización empresarial de Hidroeléctrica Española permite estimar dos funciones de costes por separado con un único producto cada una, por lo que si existen economías de escala se podrá considerar que es un monopolio natural. Hidroeléctrica Española distribuía electricidad a alta tensión en los mercados de Madrid, Valencia, Alcoy, Alicante, Sagunto y Castellón de la Plana. Unión Eléctrica de Cartagena se encargaba de la distribución a alta y a baja tensión en Cartagena.

Las filiales y otras empresas se encargaban de la distribución a baja tensión en dichos centros de consumo. En este estudio se ha escogido la Cooperativa Electra Madrid, que era distribuidora de Hidroeléctrica Española en Madrid.

${ }^{14}$ Bowers (1982), pp. 175-177. 
La red de la Cooperativa Electra Madrid distribuía electricidad en corriente continua. La red de corriente alterna se empezó a extender a partir de 1927, aunque en 1932 su importancia era aún pequeña. El cambio de continua a alterna permitía realizar grandes economías a las empresas distribuidoras. El ahorro en pérdidas de transformación ascendía a un 20 por cien, al que habría que añadir la disminución en las pérdidas de transporte y un aumento del consumo por ser suministrada a un voltaje superior. La existencia de dos redes significa que se contaba con dos productos bien diferenciados, kilovatios en corriente continua y kilovatios en corriente alterna, que precisan instalaciones diferentes para su consumo en casa de los abonados. Esto invalida la utilización de las economías de escala para determinar la condición de monopolio natural. Además, por existir cambio técnico no se podría aislar las economías de escala de las economías derivadas del cambio de tecnología. Por esta razón la estimación se ha limitado al período comprendido entre 1914 y 1927 cuando solamente existía la red de corriente continua. A pesar de que la documentación utilizada permite iniciar el estudio en 1912, se ha aplazado hasta 1914 por ser el primer año después de la guerra de precios.

\subsection{El modelo}

El trabajo pionero en el análisis de las economías de escala de la industria eléctrica fue el de Nelson (1962), que estimó las economías de escala en la fase de generación de la industria eléctrica norteamericana de 1955. Nelson estimó las economías de escala a través de una función de costes derivada de la función de producción Cobb-Douglas. Posteriormente, Christensen y Greene (1976) utilizaron una función logarítmica trascendental -translog-con los datos de 1955 y 1970. A pesar de que la estimación de una translog es deseable porque impone menos restricciones a la función de costes, el número de coeficientes a estimar es demasiado elevado dado el limitado número de observaciones de que se dispone. Así pues, se ha optado por el primer método consciente de las mayores restricciones.

La función de costes dual se obtiene de la función de producción CobbDouglas.

Siendo

$\mathrm{Q}=\mathrm{A} \mathrm{L}^{\alpha 1} \mathrm{~K}^{\alpha 2} \mathrm{y} \mathrm{C}=\mathrm{w} \mathrm{L}+\mathrm{i} \mathrm{K}$

$\mathrm{C}=\mathrm{k} \mathrm{Q}^{1 / \mathrm{r}} \mathrm{W}^{\alpha 1 / \mathrm{r}} \mathbf{i}^{\alpha 2 / \mathrm{r}}$

donde

$k=r\left(A \alpha_{1}^{\alpha 1} \alpha_{2}^{\alpha 2}\right)^{-1 / r}$

se aplican logaritmos para linealizar la función de costes:

$\ln C=\ln \mathrm{k}+1 / \mathrm{r} \ln \mathrm{Q}+\alpha_{1} / \mathrm{r} \ln \mathrm{w}+\alpha_{2} / \mathrm{r} \operatorname{lni}$

La función de costes a estimar en cada fase de producción es:

$\ln C=\beta_{0}+\beta_{q} \ln Q+\beta_{w} \ln w+\beta_{i} \operatorname{lni}$ 
donde C es el coste total, Q la producción, w son los salarios e i es el precio del capital. Esta función tiene la ventaja de que el coeficiente de la producción es la inversa de los rendimientos a escala. Las economías de escala pueden definirse como:

$$
\mathrm{ECE}=1-\mathrm{r}
$$

\subsection{Los datos}

Los datos utilizados para estimar las funciones de coste de las fases de generación, transporte y distribución a alta tensión proceden de Resúmenes de explotación y establecimiento por instalaciones para los años 1914-1935, que se encuentran en el Archivo Iberdrola Salto de Alcántara:

- Función de generación hidroeléctrica. En la función de costes de generación hidroeléctrica, el coste total es la suma de los gastos de administración y producción que la empresa especifica para cada central más el coste de capital. El coste del capital se ha calculado con los datos de establecimiento por centrales de la empresa y el precio del capital. Gracias a que los datos de establecimiento aparecen desagregados, se ha considerado el capital de la central desde el primer año completo de funcionamiento. El coste total se ha deflactado utilizando el índice de precios al por mayor ${ }^{15}$.

El precio del capital se ha determinado a partir del coste del capital propio más el coste del capital ajeno dividido por el volumen de acciones más obligaciones en circulación. El coste del capital propio, entendido como el rendimiento esperado por los accionistas al hacer la inversión, se ha calculado utilizando los dividendos repartidos, siempre y cuando éstos no fueran inferiores al rendimiento del capital ajeno. El coste del capital ajeno es el pago anual de intereses a los obligacionistas.

Como producción se entiende la electricidad generada por las centrales desde el primer año completo de funcionamiento.

Los salarios corresponden al salario unitario calculado a partir de los gastos de personal de las centrales, abarcando tanto al personal administrativo como al de producción. Esta cantidad se ha deflactado utilizando el mismo deflactor que para el coste total. Dado que solamente se dispone del número de trabajadores por central de 1935, se ha supuesto que el número de trabajadores no varió desde la entrada en funcionamiento de la misma. Este supuesto es factible porque no hubo un cambio tecnológico significativo en las centrales que ahorrara trabajo.

- Función de transporte. El coste total es la suma de los gastos correspondientes a las líneas de transporte más el coste de capital. Para obtener el capital se

\footnotetext{
15 Jordi Maluquer de Motes (1989), p.
} 
han agregado las cantidades correspondientes a los apartados de líneas de transporte, compuesto por líneas de transporte y líneas secundarias, y de las centrales de recepción y transformación de la cuenta de establecimiento. El precio del capital es el mismo que el utilizado en la función de la fase de generación.

La producción se refiere a la hidroelectricidad generada desde que la central entra en funcionamiento. Los salarios se han calculado de la misma forma que en generación, utilizando los gastos de personal correspondientes a las líneas de transporte y recepción y transformación.

El coste total y los salarios se han deflactado por el mismo método que los datos de la fase de generación.

- Función de distribución a alta tensión. El coste total es el resultado de sumar los gastos correspondientes a distribución, que incluyen los gastos de personal, explotación y conservación, más el coste de capital. El precio del capital es el utilizado en generación. El coste total se ha deflactado de la misma forma que en la fase de generación.

El dato de producción corresponde a la electricidad consumida por los abonados industriales. Dado que solamente se dispone de este dato para los últimos años del período estudiado, el consumo industrial se ha obtenido a partir del consumo anual en cada plaza, aplicando el porcentaje que representaba el consumo industrial en cada una de ellas en 1935. De esta forma se excluye la electricidad destinada a las distribuidoras, que se realizaba a través de las líneas secundarias. El consumo de Cartagena no se ha incluido porque Hidroeléctrica Española no distribuía a alta tensión en aquella zona.

Dado que no se conoce el número de trabajadores dedicados a esta fase, se ha utilizado el salario obtenido para la fase de transporte.

- Función de distribución a baja tensión. Los datos de la Cooperativa Electra Madrid proceden de la Estadística anual desde la constitución de la sociedad, 1910-1932.

El coste total se ha hallado a partir del gasto total de explotación de la estadística, al que se ha añadido el coste del capital. El gasto total de explotación incluye, entre otros, los gastos de personal, pagos al productor e impuestos. Es necesario aclarar que Electra no pagaba un precio por kilovatio recibido de Hidroeléctrica Española, sino que entregaba «el 50 por ciento de los sobrantes líquidos ingresos por venta menos gastos de explotación, deducciones estatutarias y el porcentaje destinado a fondo de reversión-» ${ }^{16}$. Dado que los pagos al productor

${ }^{16}$ Escritura de suministro de fluido eléctrico a 4 de abril de 1910. Archivo Iberdrola, sede central, carp. 10007-1 
no están en relación con los kilovatios recibidos sino con los beneficios obtenidos, se ha procedido a realizar dos estimaciones de la función de costes. Una donde los pagos al productor son las cantidades que recibió Hidroeléctrica Española y otra donde éstos han sido sustituidos por la cantidad que Electra hubiera pagado por los kilovatios recibidos de haber sido una distribuidora independiente. El precio utilizado ha sido de 0,06 pesetas por kilovatio hora durante todo el período considerado, entre 1914 y 1927. El precio se ha mantenido constante en términos nominales, pues el precio que Unión Eléctrica Madrileña e Hidroeléctrica Española pagaron por la electricidad suministrada por el Canal de Isabel II se mantuvo a 0,05 pesetas entre 1916 y 192917. Estos supuestos son plausibles porque los contratos con los grandes abonados eran a largo plazo. En cuanto al nivel del precio, se ha optado por 0,06 ptas/kWh, considerando que se encontraría entre 0,05 y 0,08 ptas/kWh, que corresponden al precio pagado al Canal de Isabel II y a Hidráulica Santillana por Electra en 1917 respectivamente. El nivel de precio escogido genera variaciones mínimas en los coeficientes estimados, por lo que no altera los resultados.

Para obtener el coste del capital se ha utilizado el valor del inmovilizado -producción y distribución- procedente de los balances de la compañía, y el precio del capital. Para conocer este último se han ponderado los dividendos y el interés que retribuían las acciones y las obligaciones en circulación respectivamente.

La producción son los kilovatios facturados. Al utilizar estos últimos en vez de los kilovatios recibidos se incorporan las pérdidas de transformación y distribución en la función de costes de forma indirecta.

Los salarios corresponden al salario unitario, que se ha calculado a partir de los gastos de personal de Electra y el número de trabajadores. Dado que no se dispone de la cifra anual de trabajadores se ha estimado con el dato de $1930 \mathrm{y}$ el número de kilovatios recibidos.

\subsection{Los resultados}

Las distintas ecuaciones se han estimado utilizando el método de mínimos cuadráticos ordinarios. Las estimaciones de las ecuaciones de transporte y distribución a alta tensión proporcionan correlación directa. En ambos casos se procedió a aplicar el procedimiento iterativo de Cochrane-Orcutt para eliminar la correlación de primer grado.

El cuadro 2 presenta los resultados de la estimación correspondiente a la fase de generación. El coeficiente de determinación es alto, y el estadístico Durbin-

\footnotetext{
${ }^{17}$ En el concurso de 1916 para la adquisición de la electricidad producida por el Canal de Isabel II se ofreció el kilovatio a 0,05 ptas/kWh. Este precio estuvo vigente, por lo menos, hasta 1929.
} 
Watson permite asegurar que no existe autocorrelación positiva en los errores. Los coeficientes estimados son todos significativos y su signo se ajusta a la teoría económica.

\section{CUADRO 2}

\section{RESULTADO DE LAESTIMACIÓN DE LA FUNCIÓN DE COSTES DE LA FASE DE GENERACIÓN}

\begin{tabular}{|l|c|c|}
\hline & Coeficiente estimado & Estadístico t \\
\hline $\boldsymbol{\beta}_{\mathbf{0}}$ & $-14,60$ & $-4,76^{*}$ \\
\hline $\boldsymbol{\beta}_{\mathrm{q}}$ & 0,87 & $3,57^{*}$ \\
\hline $\boldsymbol{\beta}_{\mathrm{w}}$ & 0,56 & $2,85^{*}$ \\
\hline $\boldsymbol{\beta}_{\mathbf{i}}$ & 4,29 & $4,63^{*}$ \\
\hline $\mathbf{R}^{\mathbf{2}}$ & 0,94 & \\
\hline $\mathbf{D W}$ & $1,71\left(\mathrm{~d}_{\mathrm{u}}=1,664\right)$ & \\
\hline $\mathbf{n}$ & 22 & \\
\hline
\end{tabular}

*significativos al 0,05.

La estimación realizada permite establecer que los rendimientos a escala eran del orden de 1,15. Por lo tanto, la fase de generación presentaba economías de escala: 0,15. La presencia de economías de escala permite afirmar que la fase de generación hidroeléctrica tenía las características de monopolio natural durante el período estudiado.

El cuadro 3 muestra los resultados de la estimación de la ecuación de costes de la fase de transporte. El coeficiente de determinación es muy elevado, y el estadístico Durbin-Watson permite descartar la presencia de autocorrelación positiva de segundo orden. Los coeficientes estimados son todos significativos y su signo se ajusta a la teoría económica.

Como era de esperar, los rendimientos a escala de la fase de transporte eran crecientes -ascendían a 1,45-, y mayores que los de la fase de generación, siendo las economías de escala en transporte de 0,45. Dado que existían economías de escala y que se trataba de un único producto, se puede afirmar que la fase de transporte presentaba las características de monopolio natural entre 1914 y 1935.

Los resultados de la estimación de la ecuación de costes de distribución a alta tensión se exponen en el cuadro 4. El coeficiente de determinación es elevado, y el estadístico Durbin-Watson permite descartar la presencia de autocorrelación positiva de segundo orden. Los coeficientes estimados son todos significativos y su signo se ajusta a la teoría económica. 


\section{CUADRO 3}

\section{RESULTADO DE LA ESTIMACIÓN DE LA FUNCIÓN} DE COSTES DE LA FASE DE TRANSPORTE

\begin{tabular}{|l|c|c|}
\hline & Coeficiente estimado & Estadístico t \\
\hline $\boldsymbol{\beta}_{\mathbf{0}}$ & $-8,52$ & $-4,71^{*}$ \\
\hline $\boldsymbol{\beta}_{\mathrm{q}}$ & 0,69 & $5,23^{*}$ \\
\hline $\boldsymbol{\beta}_{\mathrm{w}}$ & 0,65 & $6,27^{*}$ \\
\hline $\boldsymbol{\beta}_{\mathbf{i}}$ & 2,42 & $6,06^{*}$ \\
\hline $\mathbf{R}^{2}$ & 0,99 & \\
\hline $\mathbf{D W}$ & $2,29\left(\mathrm{~d}_{\mathrm{u}}=1,669\right)$ & \\
\hline $\mathbf{N}$ & 21 & \\
\hline AR(1) & 0,47 & \\
\hline
\end{tabular}

* significativos al 0,05.

\section{CUADRO 4}

RESULTADO DE LA ESTIMACIÓN DE LA FUNCIÓN DE COSTES DE LA FASE DE DISTRIBUCIÓN A ALTA TENSIÓN

\begin{tabular}{|l|c|c|}
\hline & Coeficiente estimado & Estadístico t \\
\hline $\boldsymbol{\beta}_{\mathbf{0}}$ & $-8,25$ & $-4,22^{*}$ \\
\hline $\boldsymbol{\beta}_{\mathrm{q}}$ & 0,81 & $5,19^{*}$ \\
\hline $\boldsymbol{\beta}_{\mathrm{w}}$ & 0,53 & $3,26^{*}$ \\
\hline $\boldsymbol{\beta}_{\mathrm{i}}$ & 1,53 & $2,66^{*}$ \\
\hline $\mathbf{R}^{\mathbf{2}}$ & 0,97 & \\
\hline $\mathbf{D W}$ & $2,40\left(\mathrm{~d}_{\mathrm{u}}=1,669\right)$ & \\
\hline $\mathbf{n}$ & 21 & \\
\hline $\mathbf{A R}(\mathbf{1})$ & 0,46 & \\
\hline
\end{tabular}

*significativos al 0,05.

La estimación realizada muestra que los rendimientos a escala eran crecientes -1,23- y, como consecuencia, la fase de distribución a alta tensión presentaba economías de escala - 0,23. Las economías de escala se encuentran a medio camino entre las de transporte y las de generación hidroeléctrica. La presencia de eco- 
nomías de escala permite afirmar que la fase de distribución a alta tensión tenía las características de monopolio natural en el período estudiado.

Por último, el cuadro 5 reúne los resultados de las dos estimaciones de la función de costes de la fase de distribución a baja tensión. Las primeras dos columnas corresponden a los resultados de la función de costes, donde los pagos del productor son las cantidades que recibió Hidroeléctrica Española y las dos últimas columnas muestran la estimación donde se considera el pago de 0,06 ptas/ kWh por los kilovatios recibidos. En ambos casos, el coeficiente de determinación es muy elevado, y el estadístico Durbin-Watson sugiere que no existe autocorrelación positiva. El signo de todos los coeficientes se ajusta a la teoría económica y son significativos al nivel de 0,05 a excepción del coeficiente de producción de la primera ecuación, que lo es al de 0,1.

\section{CUADRO 5}

\section{RESULTADO DE LA ESTIMACIÓN DE LAFUNCIÓN DE COSTES DE LA FASE DE DISTRIBUCIÓN A BAJA TENSIÓN}

\begin{tabular}{|l|c|c|c|c|}
\hline & \multicolumn{2}{|c|}{$\mathbf{5 0 \%}$ beneficios brutos } & \multicolumn{2}{c|}{ 0,06ptas/kWh } \\
\hline & $\begin{array}{c}\text { Coeficiente } \\
\text { estimado }\end{array}$ & $\begin{array}{c}\text { Estadístico } \\
\mathbf{t}\end{array}$ & $\begin{array}{c}\text { Coeficiente } \\
\text { estimado }\end{array}$ & $\begin{array}{c}\text { Estadístico } \\
\mathbf{t}\end{array}$ \\
\hline $\boldsymbol{\beta}_{\mathbf{0}}$ & 2,84 & 1,30 & 0,15 & 0,05 \\
\hline $\boldsymbol{\beta}_{\mathbf{q}}$ & 0,30 & $2,18^{* *}$ & 0,47 & $2,85^{*}$ \\
\hline $\boldsymbol{\beta}_{\mathbf{w}}$ & 0,82 & $16,05^{*}$ & 0,89 & $14,46^{*}$ \\
\hline $\boldsymbol{\beta}_{\mathbf{i}}$ & 1,02 & $4,52^{*}$ & 0,62 & $2,31^{*}$ \\
\hline $\mathbf{R}^{2}$ & 0,99 & & 0,98 & \\
\hline $\mathbf{D W}$ & $1,84\left(\mathrm{~d}_{\Perp}=1,779\right)$ & & $1,84\left(\mathrm{~d}_{\|}=1,779\right)$ & \\
\hline $\mathbf{N}$ & 14 & & 14 & \\
\hline
\end{tabular}

${ }^{*}$ significativos al $0,05 \mathrm{y}^{* *}$ significativo al 0,1.

Las dos estimaciones confirman la existencia de economías de escala. En la primera función, las economías ascendían a 2,33, y en la segunda a 1,13. Aunque la diferencia es notable entre las dos estimaciones, lo que interesa aquí es que existían economías de escala y que éstas eran superiores a las otras fases. Si se considera que en esta fase se produce un único producto -kilovatios a baja tensión en corriente continua-, se puede afirmar que la fase de distribución a baja tensión presentaba las características de un monopolio natural.

En cambio, si se considera que se distribuía más de un producto, como podían ser los kilovatios suministrados con contratos a tanto alzado y los kilovatios su- 
ministrados con contador, la presencia de economías de escala no sería condición suficiente para considerarla monopolio natural. En este caso, haría falta analizar la subaditividad de la función de costes, lo cual no es posible dada la información disponible ${ }^{18}$.

Al discutir las economías de densidad, en el apartado 3.2, se estableció que existían economías de densidad de producto gracias al aumento del consumo por abonado. El haber demostrado la presencia de economías de escala indica que las economías de densidad de producto no fueron neutralizadas por las posibles deseconomías de tamaño o densidad de abonado que podrían haberse dado. Por lo tanto, la existencia de economías de densidad indica a su vez que la mejor forma de organizar la distribución era a través de una única empresa.

A modo de síntesis, se puede decir que existían economías de escala en todas las fases de producción, siendo las más elevadas las correspondientes a la distribución a baja tensión y al transporte. Dado que se ha considerado que en cada función estimada se generaba un único producto, se puede afirmar que la industria eléctrica del período anterior a la Guerra Civil presentaba las características de un monopolio natural. Esto llevaría a las empresas a aumentar su capacidad de producción y convertirse en monopolios. Este hecho indica que la forma de organización monopolística existente en el mercado era la óptima.

Adicionalmente, las estimaciones de las funciones de costes permiten conocer el peso del factor capital en las diferentes fases. Los resultados confirman lo apuntado en el apartado 3.1. La fase más capital intensiva era la de generación de hidroelectricidad, seguida del transporte, y la menos capital intensiva era la de distribución a baja tensión. Esta fase era la más susceptible a los cambios en los salarios, pero aun así un aumento de los salarios produciría un incremento menos que proporcional en los costes.

\section{CONCLUSIÓN}

El examen de la industria eléctrica ha demostrado el carácter de monopolio natural de ésta durante la etapa de predominio hidroeléctrico estudiada, 1914-1935. Los resultados demuestran que las tres fases del proceso de producción -generación, transporte y distribución a alta y baja tensión-, presentaban economías de escala. Así pues, la industria eléctrica era un monopolio natural porque ela-

\footnotetext{
${ }^{18}$ Salvanes y Tjotta (1998) es el único estudio publicado que analiza la subaditividad de la función de costes de la distribución de electricidad. Los autores llegan a la conclusión de que la distribución de electricidad es un monopolio natural.
} 
boraba un único producto en cada fase. Solamente en la fase de distribución a baja tensión se podría argumentar que existían dos productos -contratos a tanto alzado y contratos con contador-que generaran costes diferentes. Aun aceptando esta interpretación, la presencia de economías de densidad de producto permite afirmar que la organización óptima es la distribución de electricidad a través de una única empresa. Por lo tanto, la organización monopolística adoptada por la industria eléctrica española fue la más eficiente.

La primera implicación de este resultado es que las ineficiencias que existieron no fueron causadas por la organización monopolística sino, en todo caso, por una regulación deficiente por parte de las administraciones públicas, que no consiguieron limitar suficientemente las pérdidas de bienestar que generaba el comportamiento monopolístico de las empresas. La segunda es que en los mercados donde existía más de una empresa no se estaba produciendo de la forma más eficiente posible. La asignación de recursos pasó a ser más eficiente cuando la producción se organizó de forma monopolística. Para el consumidor no está claro cuál fue la mejor organización. Por un lado, depende del grado de ineficiencia de la regulación en forzar que los costes menores de la empresa monopolística se tradujeran en precios bajos. Por otro, depende del grado de ineficiencia productiva de las empresas en mercados compartidos, que incapacitaba a éstas para ofrecer la electricidad a los precios más bajos posibles. Esta problemática es el objetivo de una futura investigación.

\section{FUENTES}

Resúmenes de explotación y establecimiento, Carpetas de Datos Estadísticos de Hidroeléctrica Española, años 1914-1935, Archivo Iberdrola Salto de Alcántara -AISA-, Alcántara, Fondo Hidroeléctrica Española.

Estadística anual desde la constitución de la sociedad, 1910-1932, Archivo Iberdrola Salto de Alcántara -AISA-, Alcántara, Fondo filiales, Cooperativa Electra Madrid.

\section{BIBLIOGRAFÍA}

AnTolín, F. (1999): «Iniciativa privada y política pública en el desarrollo de la industria eléctrica en España. La hegemonía de la gestión privada, 1875-1950». Revista de Historia Económica XVII, pp.411-445.

Anuario Financiero y de Sociedades Anónimas (1931). Madrid.

Aubanell, A. M. (1992): «La competencia en la distribución de electricidad de Madrid». Revista de Historia Industrial 2, pp. 143-171.

- (2000): «Estrategia empresarial y estrategia financiera de la Sociedad Hidroeléctrica Española, 1907-1935». Revista de Historia Industrial 17, pp.153-185. 
Baumol, W. J. (1977): «On the Proper Cost Tests for Natural Monopoly in a Multiproduct Industry». The American Economic Review 5, pp. 809-822.

Bowers, B. (1982): A History of Electric Light and Power. Stevenage: Peter Peregrinus Ltd.

Carreras, A. y TAfunell, X. (1993): «La gran empresa en España (1917-1974). Una primera aproximación». Revista de Historia Industrial, 3, pp. 127-175.

CAYÓN, f. (2002): «Hidroeléctrica Española: un análisis de sus primeros años de actividad (1907-1936)». Revista de Historia Económica XX, pp.301-334.

Christensen, L. R. y Greene, W. H. (1976): «Economies of Scale in U.S. Electric Power Generation». Journal of Political Economy 4, pp. 655-676.

Craywood, R. (1956): Electric Utility Rate Economics. Nueva York.

Crew, M. A. y Kleindorfer, P. R. (1986): The Economics of Public Utility Regulation. Londres: Macmillan.

Garfield, P. J. y Lovejoy, W. F. (1964): Public Utility Economics. Englewood Cliffs: Prentice Hall.

Joskow, P. L. y Schmalensee, R. (1983): Markets for Power. An Analysis of Electric Utility Deregulation. Cambridge Mass: The MIT Press.

Maluquer de Motes, J. (1989): «Precios, salarios y beneficios. La distribución funcional de la renta», en A. Carreras (coord.), Estadísticas históricas de España, siglos XIX-XX. Madrid: Fundación Banco Exterior, pp. 495-532.

Nelson, R. A. y Primeaux, W. (1988): «The Effects of Competition on Transmission and Distribution Costs in the Municipal Electric Industry». Land Economics 64, pp.338-346.

Nerlove, M. (1963): «Returns to Scale in Electricity Supply», en C. F. Christ et al., Measurement in Economics. Stanford: Stanford University Press, pp. 167-198.

Primeaux, W. (1975): «A Reexamination of the Monopoly Market Structure for Electric Utilities», en A. Phillips (ed.), Promoting Competition in Regulated Markets. Washington: The Brookings Institution, pp. 175-200.

- (1979): «Some problems with natural monopoly». The Antitrust Bulletin 24, pp. 63-85.

RoberTs, M. J. (1986): «Economies of Density and Size in the Production and Delivery of Electric Power». Land Economics 62 (4), pp. 378-387.

Rushdi, A. A. (1991): «Economies of scale and factor substitution in electricity supply industry». Energy Economics 13, pp. 219-229.

Salvanes, K. G. y Tuotta, S. (1998): «A Test for Natural Monopoly with Application to Norwegian Electricity Distribution». Review of Industrial Organisation 13, pp. 669685.

Sharkey, W. W. (1982): The Theory of Natural Monopoly. Cambridge: CUP.

SING, M. (1987): «Are Combination Gas and Electricity Utilities Multiproduct Natural Monopolies?». The Review of Economics and Statistics 3, pp. 392-398.

Sociedad Hidroeléctrica Española (1931): Memoria que el Consejo de Administración presenta a la Junta General de Accionistas. Madrid.

SuDRIÀ, C. (1990): «La industria eléctrica y el desarrollo económico de España», en José L. García Delgado, Electricidad y desarrollo: perspectiva histórica de un siglo. Hidroeléctrica del Cantábrico. Madrid, pp. 147-184.

Tedde, P. (1987): «Hidroeléctrica Española: una contribución empresarial al proceso de crecimiento económico», en 75 aniversario de Hidroeléctrica Española. Madrid.

Thompson, H. G. (1997): «Cost Efficiency in Power Procurement and Delivery Services in the Electric Utility Industry». Land Economics, 73, pp. 287-296. 
Troesken, W. (1995): «The institutional Antecedents of State Utility Regulation: The Chicago Gas Industry, 1860 to 1913», en G. Libecap y C. Goldin (eds.), The regulated economy. A historical approach to political economy. Chicago: The University of Chicago, pp. 55-80.

Unión ElÉCtRICA MadRILEÑa (1931): Memoria que el Consejo de Administración de la Sociedad Unión Eléctrica Madrileña presenta a la Junta General de Accionistas. Madrid.

WeIss, L. (1975): «Antitrust in the Electric Power Industry», en A. Phillips (ed.), Promoting Competition in Regulated Markets. Washington: The Brookings Institution, pp. 175-200.

\section{APÉNDICE}

La función de costes a estimar correspondiente a la generación térmica es la misma que la utilizada en el resto de las fases:

$$
\ln C=\beta_{0}+\beta_{q} \ln Q+\beta_{w} \operatorname{lnw}+\beta_{i} \operatorname{lni}
$$

donde C es el coste total, Q la producción, w son los salarios e i es el precio del capital.

El coste total son los gastos de producción de las tres centrales térmicas -Madrid, Valencia y Cartagena-, más el coste de capital. Este último se ha calculado a partir de la cuenta de establecimiento aplicando el precio del capital utilizado en la generación hidroeléctrica y en el transporte.

La producción se refiere a la suma de la electricidad generada en las diferentes centrales. No se ha podido obtener el salario unitario de los trabajadores de las centrales térmicas porque no se dispone del número de trabajadores ocupados en cada central a lo largo del período. Dado que el número de trabajadores en las centrales térmicas podía oscilar bastante, se ha optado por utilizar los salarios de los trabajadores de la fase de generación hidroeléctrica. Por último, el precio del carbón se ha obtenido sumando las cantidades pagadas por el consumo de carbón en las diferentes centrales, divididas por las toneladas consumidas en cada central.

Los resultados, que muestra el cuadro A1, no son aceptables porque el coeficiente del precio del capital es negativo. $\mathrm{O}$ sea, cuando aumenta el precio del capital los costes disminuyen. Este resultado indica que existe un problema con los datos utilizados para estimar la función de costes. Un posible origen del problema podría estar en la gran variación anual del peso de los costes de personal, capital y carbón dentro del coste total según sea el nivel de producción. Por ejemplo, el fuerte estiaje de 1918 hizo necesario un uso elevado de las instalaciones termoeléctricas, lo cual provocó, junto al aumento del precio del carbón, que el peso de coste del carbón alcanzara el 84,43 por cien de los costes totales, mientras que el coste del capital era un 8,89 y los salarios un 2,23 por cien. Por el contrario, en 1935, cuando las instalaciones de Millares estaban en pleno funciona- 
miento y había exceso de producción hidroeléctrica, el porcentaje del coste del carbón cayó hasta el 1,81 por cien, disparándose el del coste del capital hasta el 80,43 por cien, correspondiendo el restante 12,17 por cien al coste del trabajo. Por lo que se refiere al coeficiente del producto, a pesar de que es significativo, es poco probable que fuera tan bajo y que por lo tanto la generación térmica tuviera unas economías de escala de 3,76.

\section{CUADRO A1}

\section{RESULTADO DE LA ESTIMACIÓN DE LA FUNCIÓN DE COSTES DE LA GENERACIÓN TÉRMICA}

\begin{tabular}{|l|c|c|c|c|}
\hline & \multicolumn{2}{|c|}{ 1914-1935 } & \multicolumn{2}{c|}{ Mensual 1912 a 1914 } \\
\hline & $\begin{array}{c}\text { Coeficiente } \\
\text { estimado }\end{array}$ & $\begin{array}{c}\text { Estadístico } \\
\mathbf{t}\end{array}$ & $\begin{array}{c}\text { Coeficiente } \\
\text { estimado }\end{array}$ & $\begin{array}{c}\text { Estadístico } \\
\mathbf{t}\end{array}$ \\
\hline $\boldsymbol{\beta}_{\mathbf{0}}$ & 9,40 & $4,73^{*}$ & $-1,23$ & $-1,88^{*}$ \\
\hline $\boldsymbol{\beta}_{\mathbf{q}}$ & 0,21 & $7,98^{*}$ & 0,70 & $35,17^{*}$ \\
\hline $\boldsymbol{\beta}_{\mathbf{w}}$ & 0,34 & $2,87^{*}$ & & \\
\hline $\boldsymbol{\beta}_{\mathbf{i}}$ & $-1,71$ & $-2,91^{*}$ & & \\
\hline $\boldsymbol{\beta}_{\mathbf{p . c a r b o ́ n}}$ & 0,61 & $1,84^{* *}$ & 0,78 & $4,40^{*}$ \\
\hline $\mathbf{R}^{2}$ & 0,86 & & 0,98 & \\
\hline $\mathbf{D W}$ & $2,12\left(\mathrm{~d}_{\mathrm{u}}=1,80\right)$ & & $1,99\left(\mathrm{~d}_{\mathrm{u}}=1,56\right)$ & \\
\hline $\mathbf{n}$ & 22 & & 29 & \\
\hline
\end{tabular}

*significativos al 0,05 y **significativo al 0,1.

Dados los resultados, se ha estimado una función de costes utilizando los datos mensuales de la central de Madrid desde enero de 1912 a diciembre de 1914; no se dispone de información para los meses de abril y mayo de 1914. La estimación de las economías de escala, 0,43, ofrece un escenario más plausible.

A pesar de los problemas que presenta la estimación, los resultados indican que existían economías de escala en la generación térmica. 